\title{
Suppression of tumour growth by orally administered osteopontin is accompanied by alterations in tumour blood vessels
}

\author{
S R Rittling ${ }^{*, 1}, \mathrm{P} L$ Wejse $^{2}, \mathrm{~K}$ Yagiz $^{1,3}, \mathrm{G} \mathrm{A} \mathrm{Warot}{ }^{1,4}$ and T Hui ${ }^{1}$ \\ ${ }^{1}$ The Forsyth Institute, 245 First Street, Cambridge, MA 02412, USA and ${ }^{2}$ Arla Foods Ingredients Group P/S Sønderhøj 148260 \\ Viby J, Denmark
}

Background: The integrin-binding protein osteopontin is strongly associated with tumour development, yet is an abundant dietary component as a constituent of human and bovine milk. Therefore, we tested the effect of orally administered osteopontin (o-OPN) on the development of subcutaneous tumours in mice.

Methods: Bovine milk osteopontin was administered in drinking water to tumour-bearing immune-competent mice. Tumour growth, proliferation, necrosis, apoptosis and blood vessel size and number were measured. Expression of the $\alpha_{9}$ integrin was determined.

Results: o-OPN suppressed tumour growth, increased the extent of necrosis, and induced formation of abnormally large blood vessels. Anti-OPN reactivity detected in the plasma of OPN-null mice fed OPN suggested that tumour-blocking peptides were absorbed during digestion, but the o-OPN effect was likely distinct from that of an RGD peptide. Expression of the $\alpha_{9}$ integrin was detected on both tumour cells and blood vessels. Potential active peptides from the $\alpha_{9}$ binding site of OPN were identified by mass spectrometry following in vitro digestion, and injection of these peptides suppressed tumour growth.

Conclusions: These results suggest that peptides derived from o-OPN are absorbed and interfere with tumour growth and normal vessel development. o-OPN-derived peptides that target the $\alpha_{9}$ integrin are likely involved.

\begin{abstract}
Osteopontin (OPN) was originally identified as a tumourassociated protein (Senger et al, 1983; Senger et al, 1989), and its contribution to tumour development and metastasis has been well documented (Bellahcene et al, 2008). Importantly, OPN expression is upregulated in numerous tumour types, and increased expression is frequently associated with poor prognosis. As a secreted integrin-binding protein, it is thought to function by enhancing tumour cell migration and survival and by direct signaling to tumour cells (Chakraborty et al, 2006). OPN is also expressed in many cell types in the immune system, where it has proinflammatory activities, and regulates aspects of the immune response (Rittling, 2011; Uede, 2011). The protein is present at
\end{abstract}

high levels in milk, especially human milk, where one of its functions may be to support neonatal immunity (Schack et al, 2009).

It is still unclear how these various functions of OPN overlap. For instance, the host immune response to tumours is thought to be an important determinant of tumour growth: but it is still not clear whether OPN affects tumour development through its role in immune cell function, although there is evidence from animal models that this can occur (Hsieh et al, 2011). Alternatively, OPN has been suggested to enhance tumour development through suppression of reactive nitrogen species (Denhardt and Chambers, 1994). As a milk protein, OPN is ingested and processed through the intestinal system, and it is unknown

\footnotetext{
*Correspondence: Dr SR Rittling; E-mail: srittling@forsyth.org

${ }^{3}$ Current address: Tocagen Inc., 3030 Bunker Hill Street, Suite 230, San Diego, CA 92109, USA.

${ }^{4}$ Current address: Weatherford, 16210W., Hardy Road, Houston, TX 77060, USA.
} 
whether this OPN derived from ingested milk can affect the immune status of the organism or even the development of tumours. To begin to address this question, we have asked directly whether orally administered osteopontin (o-OPN) has any effect on tumour growth in a mouse model. Surprisingly, we found that o-OPN suppresses growth of OPN-expressing tumours through a mechanism related to the stability of blood vessels, possibly involving the $\alpha_{9}$ integrin.

\section{MATERIALS AND METHODS}

Tumour development. Tumours were induced in mice using rastransformed mouse embryo fibroblasts derived from wild-type mice on a mixed C57BL6/129 S7 background, as previously described (Wu et al, 2000). 275-3-2 (OPN expressing) cells were cultured in DMEM with 3\% FBS and tested for mycoplasma: positive cells were treated with Lookout Mycoplasma Elimination Kit (Sigma, St Louis, MO, USA). B6129SF1/J female mice, 5 weeks of age, were obtained from Jackson Laboratories (Bar Harbor, ME, USA) and were equilibrated in the Forsyth animal facility under SPF conditions in ventilated racks with automatic watering for 5-7 days before use. Low-level light-at-night conditions included light from emergency hallway lighting entering the mouse room through a $120 \mathrm{~cm}^{2}$ red-tinted window in the door. Subconfluent cells in exponential growth were harvested with trypsin, washed twice with PBS and resuspended in PBS at $5 \times 10^{5} \mathrm{cells} \mathrm{ml}^{-1}$ and $0.1 \mathrm{ml}$ of cell suspension was injected subcutaneously into the right flank, one site per mouse.

Mouse treatments. All treatments were initiated 5 days after injection of tumour cells. For oral administration of OPN, mice were given water bottles containing water only or water with dissolved purified bovine osteopontin (bOPN, Lacprodan OPN-10, Arla Foods, Viby J, Denmark) at $0.3 \mathrm{mg} \mathrm{ml}^{-1}$ unless otherwise indicated. Solutions were filter sterilised and changed every other day. Cilengitide (CLG) was obtained from the Cancer Therapy Evaluation Program (CTEP) programme at NIH, dissolved in $0.9 \%$ $\mathrm{NaCl}$ at $2 \mathrm{mg} \mathrm{ml}^{-1}$ and filter sterilised. Each mouse received $0.1 \mathrm{ml}$ of CLG or vehicle only by intraperitoneal (IP) injection daily, for a final dose of $10-11 \mathrm{mg} \mathrm{kg}^{-1} \mathrm{day}^{-1}$. Synthetic peptides VAYGL, GDSVA and GDS\{pSer\}VAY or scrambled peptides KYAGSDGVL and KYAG\{pSer\}DGVL (Genscript, Piscataway, NJ, USA; 95\% purity) were dissolved in $50 \mathrm{mM} \mathrm{HCl} \mathrm{at} 10 \mathrm{mg} \mathrm{ml}^{-1}$ and lyophilised to dryness to remove residual TFA. They were then dissolved in PBS; $\mathrm{pH}$ adjusted to $6.5-7$, diluted to $3.6 \mathrm{mg} \mathrm{ml}^{-1}$ (experimental) or $5.4 \mathrm{mg} \mathrm{ml}^{-1}$ (scrambled control), mixed as appropriate and filter sterilised. Mice were injected daily IP with $0.1 \mathrm{ml}$ of peptide solution for a final dose of $18 \mathrm{mg} \mathrm{kg}^{-1}$ peptide $^{-1}$ day $^{-1}$. Tumour size was measured every other day by calipers in two directions, and tumour size was calculated as previously described (Wu et al, 2000). When the first tumour in the control group reached $20 \%$ of the mouse's body weight, all the control animals $(n=10)$ and five of the treated animals were killed. The remaining mice continued receiving treatments until one tumour reached $20 \%$ of body weight, when all mice were killed. For experiments with synthetic peptides, groups of 10 mice were used, and all animals were killed when the first tumour reached $20 \%$ of body weight. Tumours were collected, weighed and portions frozen in OCT for cryosectioning, fixed in 4\% PFA or flash frozen on liquid nitrogen. All animal procedures were conducted under high standards of animal welfare and were approved by the Forsyth IACUC.

Short-term OPN feeding. OPN-deficient mice on a mixed 129S1/ S7 background (Rittling et al, 1998), bred in-house, were used at 3 or 10 weeks of age. bOPN was dissolved at $250 \mathrm{mg} \mathrm{ml}^{-1}$ in Yoo-Hoo chocolate milk, which itself contains $24 \mu \mathrm{g} \mathrm{ml}^{-1}$ bOPN.
Mice like the taste of Yoo-Hoo and would readily drink $0.2 \mathrm{ml}$ of the solution when offered. Groups of three animals were killed at different times after administration of OPN, and plasma was collected for later analysis.

Immunohistochemistry and necrosis. For CD-31 staining, $9-\mu \mathrm{m}$ cryosections were prepared, fixed in acetone for $7 \mathrm{~min}$ and dried overnight. Sections were blocked with $5 \%$ rabbit serum, and antimouse CD-31 antibody (BD, Franklin Lakes, NJ, USA; clone MEC 13.3) at 1:50 dilution was applied. Detection was with biotinylated anti-rat antibody followed by Vector (Burlingame, CA, USA) Elite $\mathrm{ABC}$ and diaminobenzidine (DAB) (for immunohistochemistry); sections were counterstained with haematoxylin. The numbers of positively stained vessels in a $0.25 \mathrm{~mm}^{2}$ area of DAB-stained tumour sections (viewed at $\times 200$ magnification) were counted manually. Total vessel area was determined using Image J (http:// rsweb.nih.gov/il/). Briefly, images of CD-31 DAB-stained tumour sections were opened in Image $J$ and the threshold function was used to identify stained areas on each section. The threshold level was adjusted to include only the stained regions: highlighted images were compared with the original image and the shape tool was used to remove unstained areas. The analyse function was used to calculate the stained area. For measurement of blood vessels in formaldehyde-fixed, paraffin-embedded sections, antibody to von Willebrand factor (vWF, Dako, Carpenteria, CA, USA; 1:800) was used following proteinase $\mathrm{K}$ digestion, and sections were counterstained with methyl green. Areas of tumours with the maximum vessel density were photographed (Weidner et al, 1991). Total vessel numbers and total vessel area (including lumen area) was determined using Image $J$ in two sections per tumour: values for the two sections were averaged. Formaldehyde-fixed, paraffinembedded sections were used to identify macrophages using F4/80 antibody (Harlan Bioscience, Indianapolis, IN, USA); Ki67 monoclonal rabbit antibody (ThermoScientific, Waltham, MA, USA) was used to identify proliferating cells following antigen retrieval in $0.01 \mathrm{M}$ citric acid $\mathrm{pH}$ 6.0. $\alpha_{9}$ integrin was identified in cryosections using antibody 552AC (Kanayama et al, 2009) with Dylight 647 anti-Syrian hamster secondary antibody (Jackson Immunoresearch, West Grove, PA, USA); secondary antibody for CD-31 for immunofluorescence was AlexaFluor 564 anti-rat IgG. Nuclei were counterstained with Yo-Pro3 (Invitrogen, Grand Island, NY, USA). Fluorescence images were acquired using a Leica (Buffalo Grove, IL, USA) TCS SPII Spectral Confocal system at $\times 63$ magnification in sequential mode, with four frames averaged per scan. For determination of necrotic areas, sections from formaldehyde-fixed, paraffin-embedded samples were stained with $\mathrm{H} \& \mathrm{E}$, and low magnification images of the entire tumour sections were obtained. Areas of necrosis were measured using Image J and divided by the total tumour area/section to calculate percentage of necrotic area. The percentage of necrotic areas of two non-adjacent sections per tumour were averaged.

Caspase 3/7. Caspase 3/7 activity was measured using a luciferase assay (Caspase-Glo 3/7; Promega, Madison, WI, USA). Cell lysates prepared in RIPA or cell lysis buffer (Cell Signaling Technologies, Danvers, MA, USA; no. 9803, $1 \mu \mathrm{g}$ tissue powder per $\mu$ l buffer) were diluted $1: 50$ or a caspase 3 standard solution was mixed with an equal volume of reconstituted caspase-glo reagent. After $30 \mathrm{~min}$, relative light units were determined using a Polar Star Optima Plate Reader (BMG Labtech, Cary, NC, USA). Caspase 3/7 units were calculated from the standard curve and divided by total protein, determined by BCA assay.

bOPN competition ELISA and in vitro digestion. For determination of OPN accumulating in the blood of mice fed high levels of bOPN, a competition ELISA was developed. Rabbit polyclonal anti-bOPN antibody, raised against bovine milk OPN (purified IgG 
fraction, kindly provided by Esben Sorensen, Aarhus University (Schack et al, 2009)) was biotinylated using EZ-Link Biotinylation kit (Pierce, Rockford, IL, USA) according to the manufacturer's instructions. Ninety-six-well plates were coated with $5 \mu \mathrm{g} \mathrm{ml}^{-1}$ bOPN overnight at $4{ }^{\circ} \mathrm{C}$ and then blocked with $0.5 \%$ rabbit serum. Plasma samples (1:10 dilutions) or standards (2000-16.5 $\mathrm{ng} \mathrm{ml}^{-1}$ bOPN) were mixed with an equal volume of diluted biotinylated anti-bOPN and incubated with shaking overnight at $4{ }^{\circ} \mathrm{C}$. Next day, these samples were added to the coated wells and incubated at room temperature. After washing, biotinylated antibody was detected with peroxidase-labeled streptavidin (Roche, Indianapolis, IN, USA) followed by TMB substrate solution. Standard curves were constructed from log-transformed values using the Prism (La Jolla, CA, USA) GraphPad software. In vitro digestion of bOPN was performed as described (Matsui et al, 2002b): briefly, $30 \mathrm{mg} \mathrm{ml}^{-1}$ of bOPN was adjusted to $\mathrm{pH} 3.0$ and digested with $0.4 \mathrm{mg} \mathrm{ml}^{-1}$ pepsin (Sigma) for $4 \mathrm{~h}$ at $37^{\circ} \mathrm{C}$. The $\mathrm{pH}$ was then adjusted to 7.0 , and chymotrypsin and trypsin (Sigma) each at $0.2 \mathrm{~g} \mathrm{ml}^{-1}$ was added. After an additional digestion at $37^{\circ} \mathrm{C}$ for $4 \mathrm{~h}$, the enzymes were inactivated at $95^{\circ} \mathrm{C}$ for $15 \mathrm{~min}$. Analysis of digestion products was performed at the Harvard Mass Spectrometry and Proteomics Resource Laboratory, FAS Center for Systems Biology, Northwest Building, Room B247, 52 Oxford Street, Cambridge, MA, USA by microcapillary reverse-phase HPLC nano-electrospray tandem mass spectrometry ( $\mu \mathrm{LC} / \mathrm{MS} /$ MS) on a Thermo LTQ-Orbitrap mass spectrometer (ThermoFisher Scientific, Waltham, MA, USA).

qPCR was performed using a Lightcycler 480 (Roche) according to the manufacturer's recommendations. Total RNA was prepared from flash-frozen tumour tissue using a Mirvana RNA isolation kit (Invitrogen); cDNA was prepared with Transcriptor First Strand Kit (Roche). Efficiency was determined using pooled cDNAs, and the level of $\alpha_{9}$ expression was calculated as normalised to EF1A1 expression and relative to mouse liver samples as calibrator. Primers used were forward- $5^{\prime}$-TCCTGAGATTTAGCGGCACT- $3^{\prime}$ and reverse-5'-TGTTCTCGGGGAAGTCATTC- $3^{\prime}$ for mouse $\alpha_{9}$
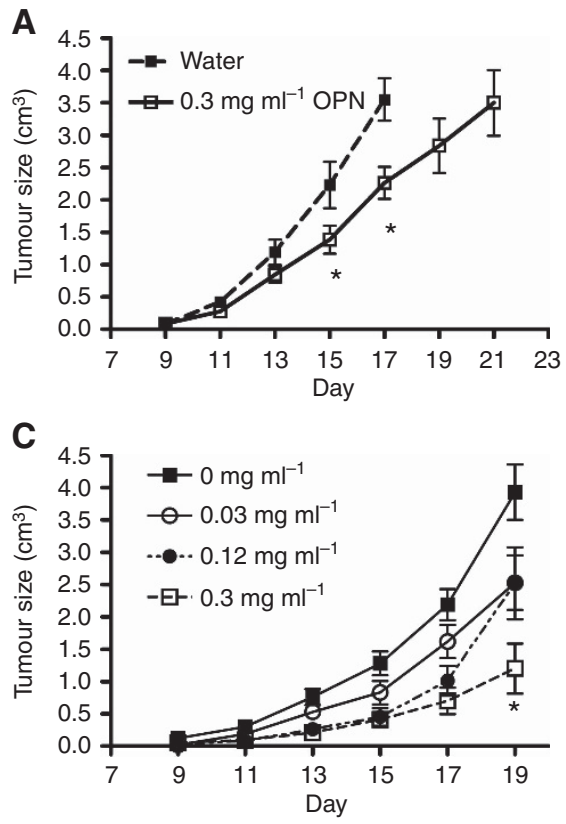

integrin and forward- $5^{\prime}$-GGAAATTCGAGACCAGCAAA- $3^{\prime}$ and reverse- $5^{\prime}$-ACACCAGCAGCAACAATCAG-3' for EF1A1.

Statistical analyses. Statistical significance was determined using the Prism software. T-tests or one-way ANOVA were used to compare treated and control samples, except for plasma OPN concentrations, which were compared using the Mann-Whitney test. All data shown are mean \pm s.e.m.

\section{RESULTS}

o-OPN suppresses tumour growth. Lacprodan OPN-10 is a purified preparation of bOPN made from cow's milk under development for use in infant formula. It is approxmately $94 \%$ OPN by protein, of which about $24 \%$ is full length $(33.9 \mathrm{kDa}$ by MALDI-TOF), with the remaining $76 \%$ being N-terminal fragments $(19.8 \mathrm{kDa}$ by MALDI-TOF), primarily cleaved by plasmin at or near the thrombin cleavage site (Christensen et al, 2010) (Esben Sorensen, personal communication). For feeding experiments, the protein was dissolved in water at $0.3 \mathrm{mg} \mathrm{ml}^{-1}$, which is the maximum concentration identified in human milk (Schack et al, 2009). Female B6129SF1 mice (6-7-weeks old) were injected on day 0 in the right flank with $5 \times 10^{4}$ 275-3-2 mouse embryo fibroblast-derived tumour cells; oral administration of OPN in the drinking water was initiated 5 days later. Growth of the tumours was significantly inhibited by o-OPN administration (Figure 1A), with an average decrease in tumour size of about $50 \%$ observed over three independent experiments on day 15 (Figure 1B). Lower doses of o-OPN were also able to reduce tumour growth in a dosedependent manner, but only the $0.3 \mathrm{mg} \mathrm{ml}^{-1}$ dose was consistently significant (Figure 1C). Mouse weight did not decrease in the first 2 days after introduction of OPN to the water, (Figure 1D) indicating that the mice are not avoiding drinking because of the presence of OPN in the water.
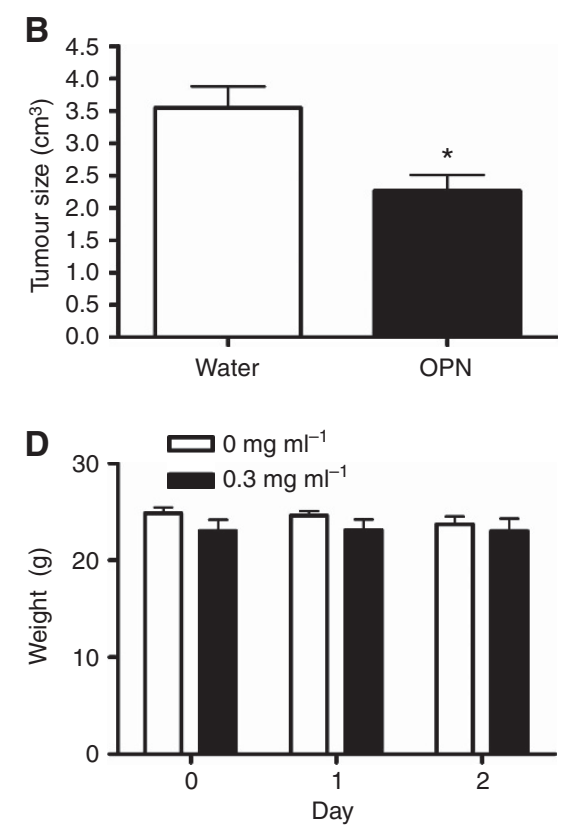

Figure 1. Effect of o-OPN on growth of 275-3-2 cells. OPN-expressing 275-3-2 cells were injected subcutaneously at $5 \times 10^{4}$ cells per mouse. OPN was added to the drinking water at the indicated concentration starting 5 days later, and tumour size was measured every other day with calipers. (A) Tumour size in control and OPN-fed mice: results of a representative experiment. $N=10$ (water); $13\left(0.3 \mathrm{mg} \mathrm{ml}{ }^{-1} \mathrm{OPN}\right.$, days 9-17) or 8 (0.3 $\mathrm{mg} \mathrm{ml}^{-1}$ OPN days 19 and 21). (B) Mean tumour size of control and OPN-fed tumours on day 15; combined results from three independent experiments. $N=30\left(0 \mathrm{mg} \mathrm{ml}^{-1} \mathrm{OPN}\right)$ or $32\left(0.3 \mathrm{mg} \mathrm{ml}^{-1} \mathrm{OPN}\right)$. (C) Tumour growth rate in mice fed different doses of OPN. (D) Mouse body weight during initiation of o-OPN feeding. Asterisk $\left(^{*}\right)$ indicates $P<0.05$ compared with control at the same time point. 
In order to begin to understand the mechanism by which o-OPN inhibits tumour growth, necrosis, proliferation and apoptosis were examined in tumours from mice fed water only (control) or $0.3 \mathrm{mg} \mathrm{ml}^{-1} \mathrm{OPN}$. Tumours were collected from mice at two different time points as described in Materials and Methods section, so these analyses compared control tumours, o-OPN d17 and o-OPN $\mathrm{d} 21$. This experimental approach allowed us to compare control and o-OPN tumours that were harvested at the same time (control and d17) and that were of the same average size (control and $\mathrm{d} 21$ ). There was a significant increase in the degree of necrosis in o-OPN d21 tumours, with some o-OPN tumours exhibiting as much as $40-50 \%$ necrosis (Figure $2 \mathrm{~A}-\mathrm{C}$ ). Note that the average size of the day $21 \mathrm{o}-\mathrm{OPN}$ tumours was similar to that of the control tumours harvested on day 17 , so the increase in necrosis was not due to differences in tumour size. It was not possible to compare necrosis in control and oOPN treated tumours on day 21, because the control tumours would have exceeded the experimental end point. On the other hand, there was no apparent effect of o-OPN on proliferation, measured as percentage of Ki67positive cells $(P=0.07$ for o-OPN d17 vs H20) or apoptosis (Figure $2 \mathrm{D}$ and $\mathrm{E})$. In both cases, there was a non-significant trend towards reduced Ki67 staining (at d17) and increased caspase 3/7 activity (at d21) in the o-OPN tumours, so that additional analyses may uncover some effect of o-OPN on these parameters. Macrophages, identified by F4/80 immunostaining, were localised predominantly at the periphery of all tumours, and there was no effect of o-OPN on their numbers (data not shown).

Peptides derived from o-OPN can be detected in the plasma of fed mice. This effect of o-OPN was quite unexpected, given that OPN is well characterised as a tumour-promoting protein (Rittling and Chambers, 2004; Bellahcene et al, 2008). Orally ingested proteins are digested by intestinal proteases and typically absorbed through enterocytes as single amino acids. However, di- and tri-peptides are also absorbed via the peptide transporter Pept1 (Adibi, 1997) and can be exported through the basolateral membrane (Charrier and Merlin, 2006). Furthermore, there is evidence for absorption of larger peptides on the basis of their biological activity (e.g., Vermeirssen et al, 2004; Cakir-Kiefer et al, 2011), and the development of food allergies may be related to absorption of antigenic peptides (Wickham et al, 2009). These observations suggest that intact OPN peptides may accumulate in the plasma of mice fed OPN. To test whether this was the case, we developed a competition ELISA for bOPN: this assay was designed to detect any peptides that can block interaction of bOPN with the polyclonal antibody used. OPN $-/-$ mice (used to avoid crossreaction with endogenous mouse OPN) were fed $50 \mathrm{mg}$ bOPN dissolved in Yoo-Hoo chocolate milk. Plasma was collected from these mice at different times after feeding and analysed using the competition ELISA: data are presented as $\mathrm{ngml}^{-1} \mathrm{bOPN}$, although the assay is probably not measuring intact bOPN (Figure 3). It is clear from these results that antibody-reactive material accumulates in the plasma of both young (3 week) and older (10 week) OPN-fed mice and that the peak of this accumulation occurs between 1 and $4 \mathrm{~h}$ after feeding. YooHoo itself contains about $24 \mu \mathrm{g} \mathrm{ml}^{-1}$ bOPN, and a minimal level of bOPN peptides could even be detected in the plasma of mice fed YooHoo only (YH, Figure 3). These data confirm that peptides derived from o-OPN can reach the circulation and suggest that the effect of o-OPN on tumour growth may be mediated by OPN peptides absorbed into the circulation.

The RGD sequence is unlikely to be the effective peptide. OPN binds to several integrins through two adjacent integrin-binding sites in the centre of the molecule: the RGD sequence and the SVVYGLR (SVAYGLK in bOPN). Of these, the RGD sequence is well known to be involved in the development of blood vessels within tumours (Brooks et al, 1994), and RGD-based compounds

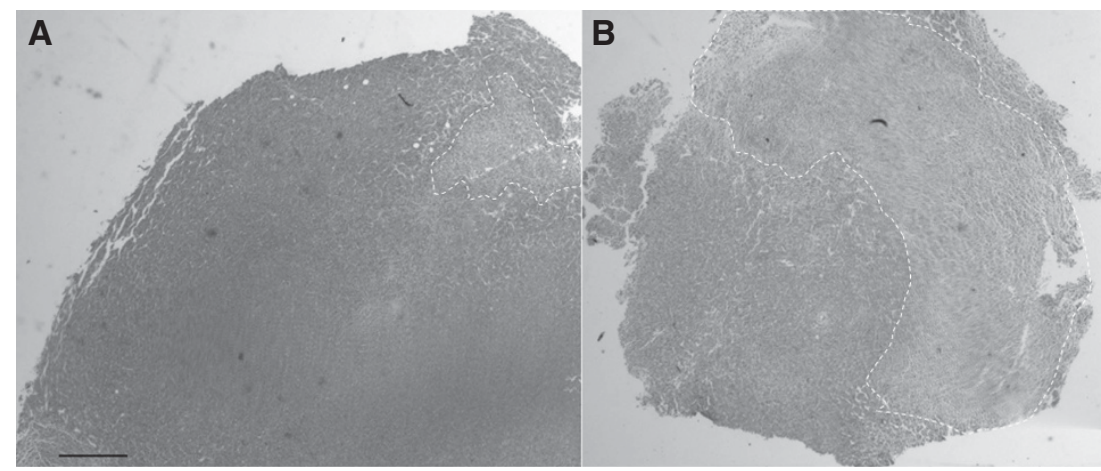

C

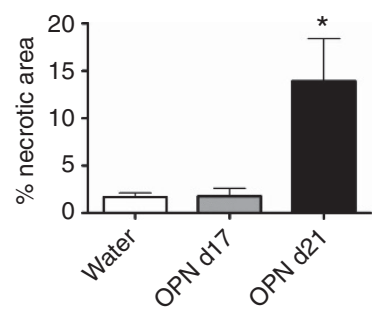

D

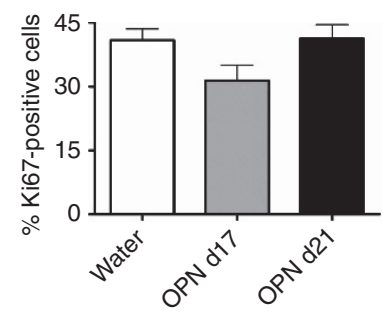

E

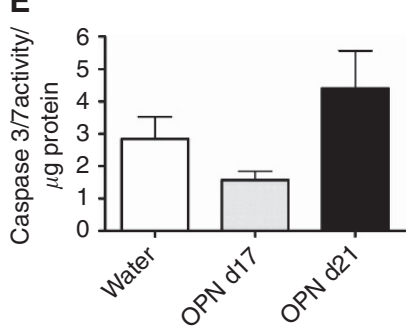

Figure 2. o-OPN induces necrosis but not growth arrest or apoptosis. (A and B) H\&E-stained sections from representative tumours showing areas of necrosis (indicated by dashed lines). (A) control tumour; (B) tumour from an o-OPN treated mouse collected on day 21 . Bar $=100 \mu \mathrm{m}$. (C) Quantification of necrotic areas in control and o-OPN tumours: OPN was administered at $0.3 \mathrm{mg} \mathrm{ml}^{-1}$, and tumours were collected on day 17 (water and OPN d17, n=10 and 5, respectively) or on day 21 (OPN d21, n=8). Necrotic areas were assessed in H\&E-stained sections, measured and expressed as a percentage of the total tumour area in the section. (D) Proliferation was assessed by Ki67 staining, and percentage of positive cells was determined by counting; groups as in panel (C). (E) Caspase 3/7 activity was determined biochemically in lysates from water- and o-OPN-treated mice; groups as in panel (C). ${ }^{\star} P<0.01$ vs water group (one-way ANOVA). 
are under investigation as chemotherapeutic, anti-angiogenic agents (Scaringi et al, 2012). We reasoned that the tumoursuppressing effect of o-OPN might be due to accumulation of its RGD peptides in the plasma of fed mice, which may then inhibit tumour growth. To test this hypothesis, the effect of o-OPN on growth of the 275-3-2 tumours was compared with that of cilengitide, a cyclic pentamer peptide containing the RGD sequence (Mas-Moruno et al, 2010). 275-3-2 cells were injected into $\mathrm{F} 1 \mathrm{mice}$ and cilengitide $\left(10 \mathrm{mg} \mathrm{kg}^{-1}\right.$, about five-fold higher dose on a molar basis as compared with o-OPN) was administered by daily IP injection starting 5 days later. As expected, cilengitide inhibited the growth of these tumours, similarly to o-OPN (Figure 4A). The mechanism of this effect appeared to be somewhat different; however, as cilengitide was effective under conditions of low-level light at night (from hallway lighting coming through a $120-\mathrm{cm}^{2}$ window), which has been previously shown to accelerate tumour growth (Dauchy et al, 2011), while o-OPN was not effective under these conditions (Figure $4 \mathrm{~A}$ and $\mathrm{B}$ ). This result

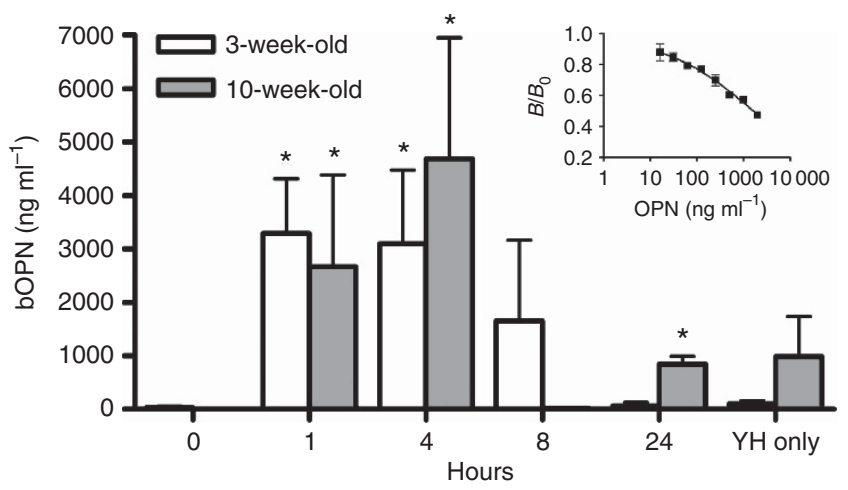

Figure 3. OPN reactivity in plasma of mice fed OPN. bOPN, $50 \mathrm{mg}$ per mouse, was dissolved in Yoo-Hoo chocolate milk (which contains bOPN) and administered orally in a bolus over a period of 15-20 min to OPN-deficient mice. Plasma was collected at different times after feeding and assayed for anti-OPN antibody reactivity using a competition ELISA. Data for both 3-week old (open bars) and 10-week old (filled bars) are shown. Inset: representative standard curve using Lacprodan-OPN10 as standard; $N=3$ per group $\mathrm{YH}=$ YooHoo. ${ }^{\star} P=0.05$ as compared with time 0 , determined by Mann-Whitney test.

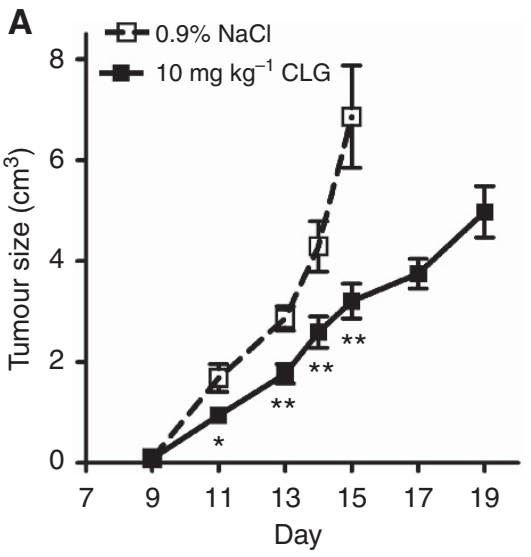

suggests that the effects of o-OPN and CLG have different mechanistic bases. The reason for the lack of effect of o-OPN under low-level light-at-night conditions is not clear, although effects on OPN expression through modification of stress hormone levels are one of several possibilities.

o-OPN regulates blood vessel size. Cilengitide is expected to affect development of blood vessels in treated tumours, and we found that the mean vessel density was decreased in CLG-treated tumours by using immunohistochemistry for CD-31 in cryosections (Figure 5E). o-OPN, on the other hand, did not affect vessel numbers in treated tumours (Figure 5A, B and E). Analysis of total vessel area revealed that o-OPN treatment led to an increase in vessel area that was significant in the $\mathrm{d} 21$ tumours (Figure 5F). As this result was unexpected, additional analyses were performed in paraffin sections of the o-OPN tumours, where blood vessels were identified using antibody to von-Willebrand factor. Areas of each tumour with the highest apparent density of blood vessels were selected (Weidner et al, 1991), and the total vessel area per field was determined. Many of the o-OPN treated tumours had regions of very large blood vessels (Figure 5D), and the total area of these vessels were significantly larger in the o-OPN-treated tumours than in controls (Figure 5G). Together, these results suggest that the effect of o-OPN may be distinct from that of RGD and that peptides derived from o-OPN may suppress tumour growth through unique effects on tumour-associated blood vessels.

$\alpha_{9}$ integrin is expressed in 275-3-2 tumours. In addition to $\alpha_{\mathrm{v}}$-containing integrins, OPN binds to the $\alpha_{4}$ and $\alpha_{9} \beta_{1}$ integrins. The $\alpha_{4}$ integrin expression is largely confined to haematopoietic and intestinal cells, and we have shown that OPN can only bind to this integrin in its fully activated state (Rittling et al, in preparation). Thus, we hypothesise that the $\alpha_{9}$ integrin may be the target of peptides derived from o-OPN, particularly as this integrin is also associated with angiogenesis and lymphangiogenesis (Hoye et al, 2012). Thus, we asked whether the $\alpha_{9}$ integrin is expressed in the 275-3-2 tumours and might be a target for peptides derived from o-OPN. qPCR demonstrated that the $\alpha_{9}$ integrin is expressed in all tumours examined at a level similar to that in normal liver, even though it is not expressed in the parental 275-3-2 cells in culture (Figure 6A). By immunofluorescence, moreover, this integrin is expressed in association with both blood vessels (Figure 6B, thin arrows) and with non-blood vessel cells,

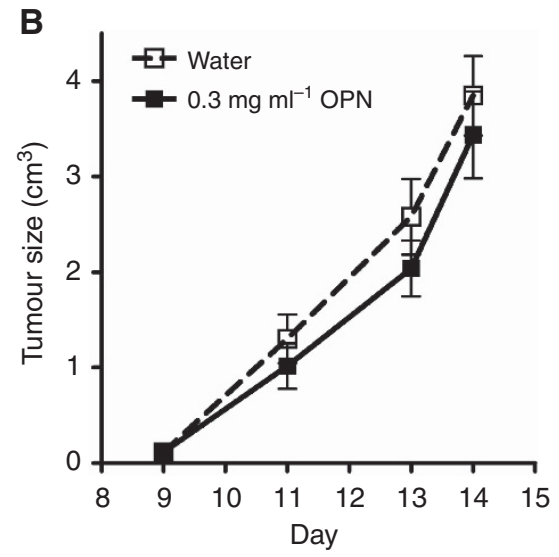

Figure 4. Cilengitide, but not OPN, suppresses tumour growth under light-at-night conditions. Mice were inoculated with 275-3-2 cells as described in Figure 1 and CLG ((A); $10 \mathrm{mg} \mathrm{kg}^{-1}$ daily by IP injection) or OPN (B) were administered starting 5 days after tumour cell inoculation; control animals received vehicle $(0.9 \% \mathrm{NaCl}$ or water) only. (A) Tumour sizes on different days in $0.9 \% \mathrm{NaCl}$ or CLG-treated mice. $n=10$ (NaCl); 13 , (CLG days 9-15); and 8, (CLG, days 17 and 19). ${ }^{\star} P<0.05 ;{ }^{\star \star} P<0.01$ compared with $\mathrm{NaCl}$. (B) Tumour sizes on different days in o-OPN ( $n=13$ ) or water $(n=10)$ treated mice. Mice were housed under low levels of light-at-night conditions as described in Materials and Methods section. 

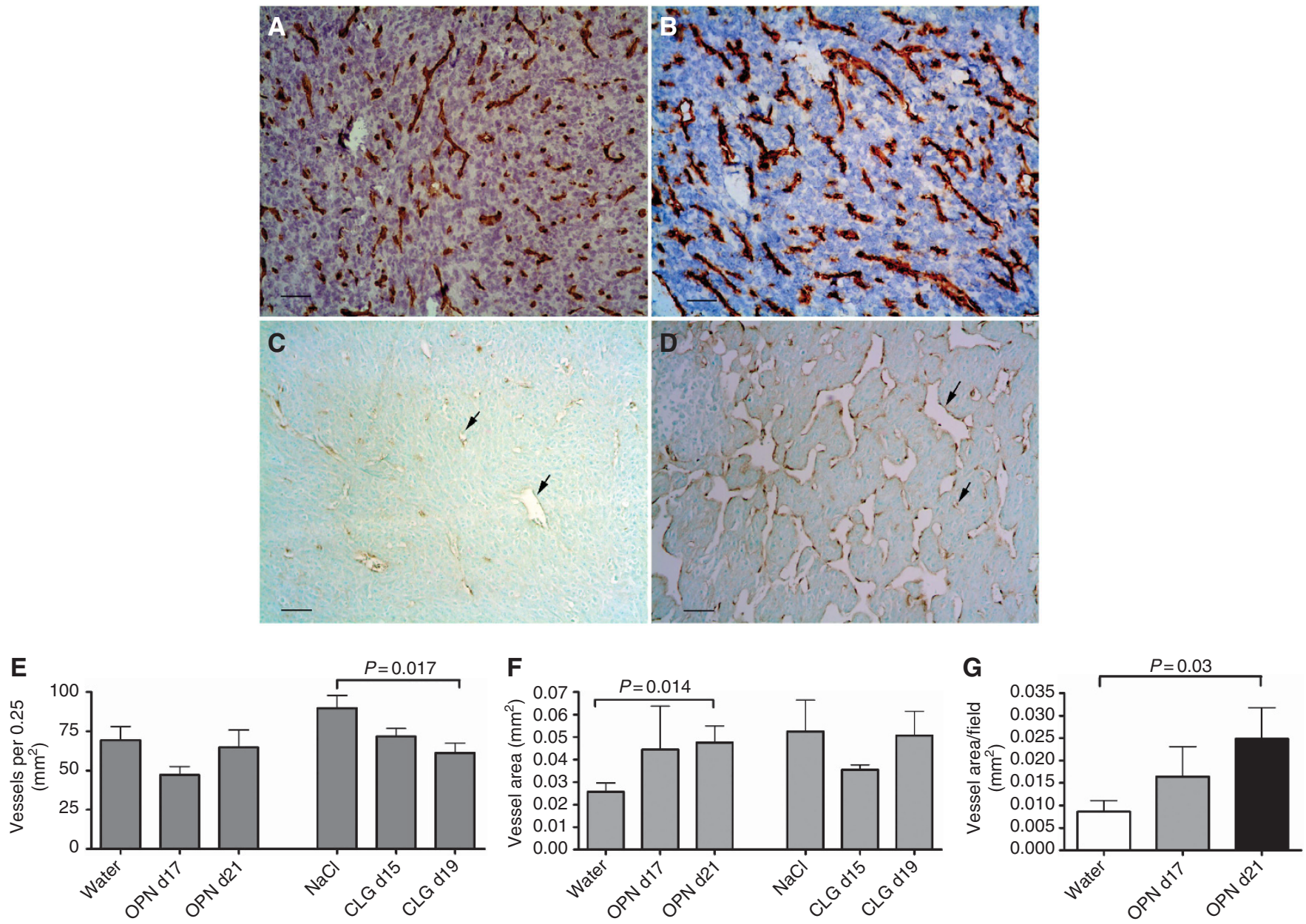

Figure 5. Analysis of vessel density in control and treated tumours. Tumours from control, o-OPN (dark-at-night conditions) or CLG (light-at-night conditions) treated mice were collected and cryosections or paraffin sections were prepared. Blood vessels were visualised with anti-CD-31 antibody or anti vWF antibody. (A and B) Cryosections from control (A) or o-OPN-treated (B) tumours were reacted with anti-CD-31 and visualised by DAB staining; sections were counterstained with haematoxylin. ( $C$ and $\mathbf{D})$ Paraffin sections from control (C) and o-OPN (D) treated tumours were reacted with anti-vWF and visualised by DAB staining. Sections were counterstained with methyl green. Arrows indicate representative large and small vessels; bar $=50 \mu \mathrm{m}$. (E) The number of CD-31-stained blood vessels in control, o-OPN- or CLG-treated tumours was manually counted in an area of $25 \mu \mathrm{m}^{2}$. Tumours were collected on day $15(\mathrm{NaCl}, n=10$ and CLG d15, n=5) or on day 19 (CLG day 19, $n=8)$ after inoculation. o-OPN groups as in Figure 2. (F) The total CD-31-stained area per section was measured using a thresholding technique in Image J. Groups as in panel (E). (G) Total vessel area in the maximally stained field of each tumour section was measured in vWF-stained sections by using Image $\mathrm{J}$ to outline blood vessels. Groups as in Figure 2.

presumably tumour cells themselves (block arrows). These results suggest that the $\alpha_{9}$ integrin is a potential target of o-OPN, and may be upregulated in tumour cells within the tumours, implying its importance to the development of these tumours.

Peptides derived from the alpha9 binding sequence of bOPN suppress tumour growth. To begin to assess the peptides that may be mediating the effects of o-OPN, we performed proteolysis in vitro of bOPN with three prominent digestive enzymes: pepsin, trypsin, and chymotrypsin. The products of this digestion were analysed by $\mu \mathrm{LC} / \mathrm{MS} / \mathrm{MS}$, and all peptides ( $>300$ Daltons) derived from bOPN were identified. Trypsin is known to cleave OPN within the RGD sequence (R/GD; (Christensen et al, 2010)), and accordingly no short peptides ( $<15$ amino acids) containing the RGD sequence were observed. On the other hand, several short peptides were identified that contained part of the SVAYGLK sequence that mediates binding to the $\alpha_{4}$ and $\alpha_{9}$ integrins (Figure 7A). Three of the identified short peptides derived from the SVAYGLK sequence: VAYGL, GDSVA, and GDS*VAY (phosphorylated on serine) were synthesised and injected together into tumour-bearing mice at a dose of
$18 \mathrm{mg} \mathrm{kg}^{-1}$ peptide $^{-1}$ day $^{-1}$ daily, starting on day 5 after tumour cell injection. Remarkably, this combination of peptides was able to suppress tumour growth similarly to o-OPN (Figure 7B), providing further support for the hypothesis that peptides derived from o-OPN are absorbed from the circulation and inhibit tumour-promoting interactions between tumourproduced OPN and the $\alpha_{9}$ integrin.

\section{DISCUSSION}

Here we describe an unanticipated effect of o-OPN in suppressing tumour growth. This effect was reproducible over three independent experiments, with an average decrease of $50 \%$ in the size of the tumours treated with o-OPN. It is unlikely that these effects of OPN are due to non-specific or indirect effects of o-OPN. The OPN preparation used here is prepared from normal cow's milk, has been tested extensively for safety and is being used as an additive for infant formula at the same concentrations used here (Peter Wejse, unpublished data). Despite this high level of safety 

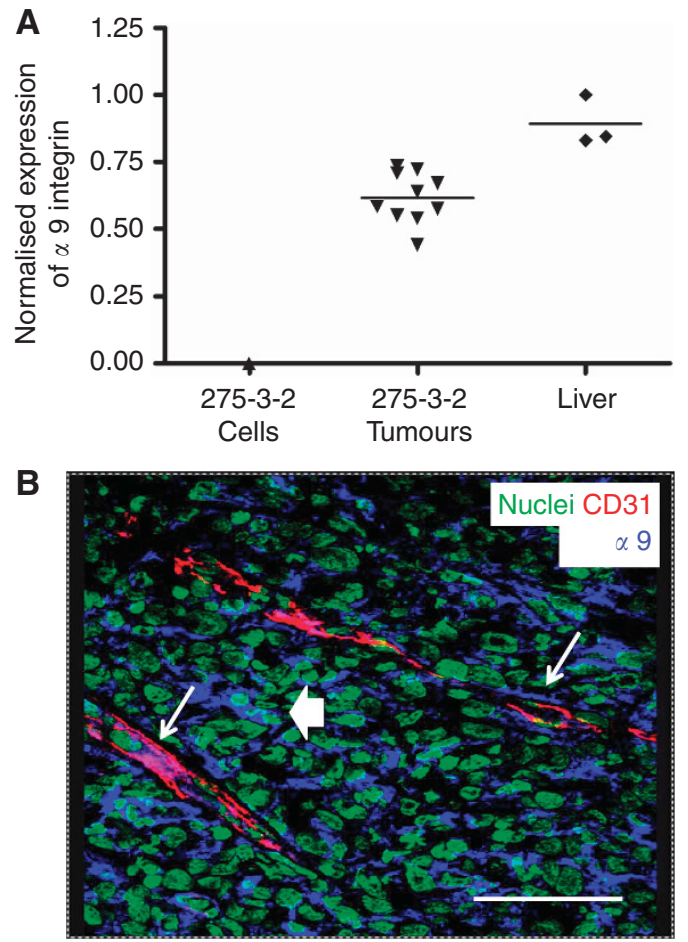

Figure 6. $\alpha_{9}$ Integrin is expressed in 275-3-2 tumours. (A) qPCR. RNA from 275-3-2 cells, several 275-3-2 tumours or from normal liver (positive control) was purified and cDNA prepared. qPCR was performed as described in Materials and Methods section and normalised to EF1a1. (B) Immunofluorescence was performed on cryosections from untreated 275-3-2 tumours for $\alpha_{9}$ integrin (blue) and CD-31 (red). Nuclei are stained green with Yo-Pro. Thin arrows indicate $\alpha_{9}$ expression closely associated with blood vessels; block arrow indicates $\alpha_{9}$ expression in non-vascular cells. Bar $=50 \mu \mathrm{m}$.

and palatability, mice are frequently neo-phobic, and it is possible that they will be reluctant to drink the OPN-containing water. Several observations suggest that this is not the case: (1) there is no effect of the compound on mouse weight; (2) we observed that the effect of o-OPN on 275-3-2 tumours is lost under conditions of low-level light-at night conditions; and (3) there is no effect of o-OPN on the growth of an OPN-deficient tumour cell line (data not shown). Together, these results suggest that the effect of o-OPN on tumour growth is not due to non-specific or indirect effects; rather that o-OPN directly suppresses tumour growth.

Our data are consistent with the idea that peptide fragments of orally ingested OPN reach the circulatory system and can directly interact with tumour or stromal cells. We and others (da Silva et al, 2009) have shown that oral administration of OPN causes the appearance of substances in the plasma that inhibit reaction of anti-OPN antibodies with intact OPN: these are most likely OPN fragments or peptides. Although the absorption of peptides through the intestinal system has been controversial, there is considerable data demonstrating that this occurs regularly and is actually a primary means of intestinal absorption of amino acids. Proteins in the small intestine are digested by the gastric and pancreatic enzymes pepsin, trypsin and chymotrypsin into small peptides (Zaloga and Siddiqui, 2004). Di- and tri-peptides are efficiently absorbed into the apical surface of intestinal epithelial cells via the Pept1 transporter (Adibi, 1997); many of these peptides are digested to amino acids within the epithelium, but secretion of these peptides through the basolateral surface is not as well characterised or understood. However, there are several studies in the literature demonstrating the appearance of di- and
A

\begin{tabular}{|l|c|c|}
\hline \multicolumn{1}{|c|}{ Peptide } & Aa \# (length) & $\begin{array}{c}\text { Times } \\
\text { identified }\end{array}$ \\
\hline RGD peptides & & \\
\hline PFIPTESANDGRGDS & $141-155(15)$ & 1 \\
\hline PFIPTESANDGRGDSVA & $141-157(17)$ & 2 \\
\hline IPTESANDGRGDSVA & $143-157(15)$ & 1 \\
\hline SVAYGLK peptides & & \\
\hline GDSVA & $153-157(5)$ & 1 \\
\hline GDSVAY & $153-158(6)$ & 2 \\
\hline GDS*VAY & $153-158(6)$ & 1 \\
\hline VAYGL & $156-160(5)$ & 2 \\
\hline
\end{tabular}

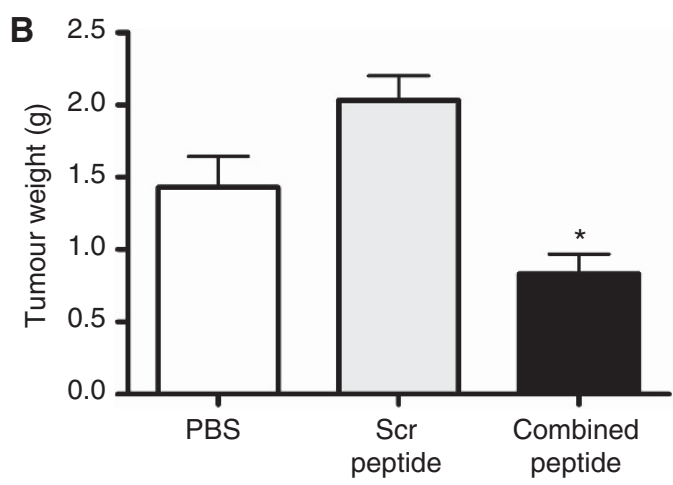

Figure 7. Peptides from bOPN suppress tumour growth. (A) Bovine OPN was digested sequentially with pepsin, trypsin and chymotrypsin as described in Materials and Methods section. All peptides $>300$ Daltons derived from bOPN in these mixtures were identified by $\mu \mathrm{LC} / \mathrm{MS} / \mathrm{MS}$. Only those peptides containing the RGD or parts of the SVAYGLK sequence are listed. *-phosphorylated on serine, Aa-amino acid. (B) OPN-derived peptides from the $\alpha_{9}$ binding site suppress the growth of 275-3-2 tumours. Synthetic peptides were dissolved in PBS and injected daily into tumour-bearing mice as in Figure 4. Scrambled peptide was a combination of KYAGSDGVL and KYAG\{pSer\}DGVL, each at $27 \mathrm{mg} \mathrm{kg}^{-1}$ day $^{-1}$; combined peptide is VAYGL, GDSVA and GDS\{pSer\}VAY, each at $18 \mathrm{mg} \mathrm{kg}^{-1}$; total peptide dose in each case was $54 \mathrm{mg} \mathrm{kg}^{-1}$ day $^{-1}$. Mice were killed on day 19, and tumours were excised and weighed. ${ }^{\star} P<0.03$ compared with PBS or scrambled peptide.

tri-peptides and longer peptides in the plasma following oral ingestion (Matsui et al, 2002a; Foltz et al, 2007) and that enterally administered peptides such as thyrotropin-stimulating hormone (a tri-peptide) and LHRH (a 10 amino-acid peptide) have biological activity (Roberts et al, 1999; Vermeirssen et al, 2004). Some peptide-based therapeutics are administered orally such as cyclosporin and desmopressin (Renukuntla et al, 2013), providing a precedent for intestinal absorption of peptides.

Our observation that o-OPN has biological activity may result from a combination of factors. First, degradation of the protein during intestinal digestion is probably required for the release of bioactive peptide, so conservation of the structure of the protein is not required. In addition, milk OPN has been shown to be relatively resistant to digestion by gastric proteases (Schack et al, 2009), possibly due to its acidic nature and posttranslational modifications. Thus, the availability of bioactive peptides from o-OPN may result from a unique confluence of events.

OPN contains the well-known tri-peptide RGD, which interacts with several integrins, including the $\alpha_{\mathrm{v}} \beta_{3}$. This integrin is preferentially expressed on developing blood vessels and is implicated in the development of tumour-associated blood vessels (Brooks et al, 1994; Weis and Cheresh, 2011). Several RGDcontaining compounds have shown anti-tumour effects in animal 
and human models (Yamada et al, 2006; Reardon et al, 2008). Thus, the RGD is a potential bioactive tri-peptide derived from o-OPN. However, the differing effects of low-level light at night on the effect of o-OPN and CLG suggest that their mechanism of action is not the same. In addition, the RGD sequence in OPN is known to be cleaved by the digestive enzyme trypsin, and we were unable to identify short peptides containing the RGD after sequential digestion of OPN with pepsin, trypsin and chymotrypsin. Taken together, these results suggest that the RGD tri-peptide is not the only bioactive compound derived from o-OPN.

bOPN contains an additional conserved sequence SVAYGLK (SVVYGLR in human) that interacts with a series of integrins, including both $\alpha_{4} \beta_{1}$ and $\alpha_{9} \beta_{1}$ (Smith et al, 1996; Yokosaki et al, 2005). Interestingly, the SVVYGLR peptide itself has been shown to enhance angiogenesis in both in vivo and in vitro models (Hamada et al, 2003, 2007; Chan et al, 2011). It is unknown whether the SVAYGLK peptide is able to reach the circulation intact; however, our in vitro digestion demonstrates that several short peptides derived from this sequence are generated during digestion. We demonstrated that a combination of three of these peptides have anti-tumour effects in the 275-3-2 tumours, when injected in combination IP, providing extremely strong support to the hypothesis that peptides derived from this sequence are antitumourigenic in our system. Three peptides were injected together to maximise the possibility of identifying bioactive peptides; whether one or all of these peptides are individually active and what is the optimal peptide for suppression of tumour growth is under active investigation. Alternatively, an epitope in the $\mathrm{N}$-terminal end of human OPN has bioactivity (Fan et al, 2008), and it is possible that peptides derived from this sequence are important in the effects of o-OPN; however, the ligand(s) for these sequences are still unknown.

Our results suggest that the mechanism of the effect of o-OPN on tumour growth is related to angiogenesis. We demonstrated that while the total number of blood vessels is not altered by o-OPN, the overall area of blood vessels is actually increased (Figure 5). This is because of an increase in the number of tumours with very large blood vessels, resembling blood sinuses that are frequently found near areas of necrosis (Figure 5D). This may suggest that these vessels are inherently unstable, or that they are inefficient at nutrient transfer, perhaps because of sluggish blood flow. Anti-angiogenesis therapy has frequently been shown to cause 'normalisation' of tumour blood vessels, resulting in increased association with pericytes and increased permeability (Weisshardt et al, 2012), but the very large vessels we observed do not have a normal appearance: additional experiments are required to understand the development and role of these structures. Nevertheless, the documented involvement of two OPN-binding integrins, $\alpha_{\mathrm{v}} \beta_{3}$ and $\alpha_{9} \beta_{1}$, in tumour-associated or normal blood vessel development provides a likely mechanistic basis for our results. Although the $\alpha_{\mathrm{v}} \beta_{3}$ is associated with neovascularisation (Niland and Eble, 2011), the $\alpha_{9} \beta_{1}$ is expressed on and required for proper formation of the lymphatic endothelium (Huang et al, 2000), and is also expressed on blood vessels, for example, in lung tissue (Staniszewska et al, 2007). The $\alpha_{9} \beta_{1}$ is a receptor for the angiogenic growth factor VEGF-A and promotes its angiogenic function (Vlahakis et al, 2007), while interaction of this integrin with its ligands thrombospondin (Staniszewska et al, 2007) or NGF (Walsh et al, 2012) mediates angiogenesis. There is also evidence for a direct effect of the $\alpha_{9}$ integrin on tumour cell invasiveness. In a model of human breast cancer, where $\alpha_{9} \beta_{1}$ expression is associated with increased metastasis (Vantyghem et al, 2005), downregulation of this integrin severely restricts tumour growth and metastasis (Majumder et al, 2012). Also in rhabdomyoma cells, $\alpha_{9}$ function is associated with increased migration and invasiveness (Masia et al, 2012). Our observation that the $\alpha_{9}$ integrin is apparently upregulated in tumour cells during tumour development may also indicate the importance of this integrin in tumourigenesis. Inhibition of $\alpha_{9} \beta_{1}$ signaling by peptides derived from o-OPN may have unique effects on vessel remodeling and/or a direct effect on tumour growth. Further work will be required to elucidate the details of these mechanisms.

In summary, our results suggest that peptides derived from o-OPN can regulate blood vessel formation in tumours so as to suppress tumour growth and that these effects may be mediated by inhibition of $\alpha_{9} \beta_{1}$ integrin function within tumours. o-OPN has been shown to ameliorate DSS-induced colitis (da Silva et al, 2009) and alcohol-induced liver damage ( $\mathrm{Ge}$ et $\mathrm{al}$, 2013), but the mechanism of both these actions are postulated to be within the digestive system. Ours is the first demonstration of an effect of o-OPN at distant sites and the first demonstration that the $\alpha_{9}$ integrin may be involved. Elucidation of the molecular mechanisms of these effects will provide new insights into the development of tumours and potential therapeutic approaches. Additionally, the safe natural product Lacprodan OPN-10 (the bOPN product used here) as a dietary additive might be an effective way to suppress tumour growth.

\section{ACKNOWLEDGEMENTS}

We thank Esben Sorensen for the gift of anti-OPN antibodies, Toshimitsu Uede for the gift of the $\alpha_{9}$ antibody and Simon Goodman for critical reading of the manuscript. The Cilengitide was generously provided by Merck KgaA and the CTEP, National Cancer Institute, NIH. We appreciate technical assistance by Hannah McLeod, Eva Xia and Carolina Brettler. This work was supported by grants from Arla Foods Ingredients.

\section{REFERENCES}

Adibi SA (1997) The oligopeptide transporter (Pept-1) in human intestine: biology and function. Gastroenterology 113(1): 332-340.

Bellahcene A, Castronovo V, Ogbureke KUE, Fisher LW, Fedarko NS (2008) Small integrin-binding ligand $\mathrm{N}$-linked glycoproteins (SIBLINGs): multifunctional proteins in cancer. Nat Rev Cancer 8(3): 212-226.

Brooks PC, Clark RA, Cheresh DA (1994) Requirement of vascular integrin alpha v beta 3 for angiogenesis. Science 264(5158): 569-571.

Cakir-Kiefer C, Le Roux Y, Balandras F, Trabalon M, Dary A, Laurent F, Gaillard JL, Miclo L (2011) In vitro digestibility of alpha-casozepine, a benzodiazepine-like peptide from bovine casein, and biological activity of its main proteolytic fragment. J Agric Food Chem 59(9): 4464-4472.

Chakraborty G, Jain S, Behera R, Ahmed M, Sharma P, Kumar V, Kundu GC (2006) The multifaceted roles of osteopontin in cell signaling, tumor progression and angiogenesis. Curr Mol Med 6(8): 819-830.

Chan LY, Gunasekera S, Henriques ST, Worth NF, Le SJ, Clark RJ, Campbell JH, Craik DJ, Daly NL (2011) Engineering pro-angiogenic peptides using stable disulfide-rich cyclic scaffolds. Blood 118(25): 6709-6717.

Charrier L, Merlin D (2006) The oligopeptide transporter hPepT1: gateway to the innate immune response. Lab Invest 86(6): 538-546.

Christensen B, Schack L, Klaning E, Sorensen ES (2010) Osteopontin is cleaved at multiple sites close to its integrin-binding motifs in milk and is a novel substrate for plasmin and cathepsin D. J Biol Chem 285(11): 7929-7937.

da Silva AP, Ellen RP, Sorensen ES, Goldberg HA, Zohar R, Sodek J (2009) Osteopontin attenuation of dextran sulfate sodium-induced colitis in mice. Lab Invest 89(10): 1169-1181.

Dauchy RT, Dupepe LM, Ooms TG, Dauchy EM, Hill CR, Mao L, Belancio VP, Slakey LM, Hill SM, Blask DE (2011) Eliminating animal facility light-at-night contamination and its effect on circadian regulation of rodent physiology, tumor growth, and metabolism: a challenge in the relocation of a cancer research laboratory. J Am Assoc Lab Anim Sci 50(3): $326-336$. 
Denhardt DT, Chambers AF (1994) Overcoming obstacles to metastasis—defenses against host defenses: osteopontin (OPN) as a shield against attack by cytotoxic host cells. J Cell Biochem 56(1): 48-51.

Fan K, Dai J, Wang H, Wei H, Cao Z, Hou S, Qian W, Li B, Zhao J, Xu H, Yang C, Guo Y (2008) Treatment of collagen-induced arthritis with an anti-osteopontin monoclonal antibody through promotion of apoptosis of both murine and human activated T cells. Arthritis Rheum 58(7): 2041-2052.

Foltz M, Meynen EE, Bianco V, van Platerink C, Koning TM, Kloek J (2007) Angiotensin converting enzyme inhibitory peptides from a lactotripeptide-enriched milk beverage are absorbed intact into the circulation. J Nutr 137(4): 953-958.

Ge X, Lu Y, Leung TM, Sorensen ES, Nieto N (2013) Milk osteopontin, a nutritional approach to prevent alcohol-induced liver injury. Am J Physiol Gastrointest Liver Physiol 304(10): G929-G939.

Hamada Y, Egusa H, Kaneda Y, Hirata I, Kawaguchi N, Hirao T, Matsumoto T, Yao M, Daito K, Suzuki M, Yatani H, Daito M, Okazaki M, Matsuura N (2007) Synthetic osteopontin-derived peptide SVVYGLR can induce neovascularization in artificial bone marrow scaffold biomaterials. Dent Mater J 26(4): 487-492.

Hamada Y, Nokihara K, Okazaki M, Fujitani W, Matsumoto T, Matsuo M, Umakoshi Y, Takahashi J, Matsuura N (2003) Angiogenic activity of osteopontin-derived peptide SVVYGLR. Biochem Biophys Res Commun 310(1): 153-157.

Hoye AM, Couchman JR, Wewer UM, Fukami K, Yoneda A (2012) The newcomer in the integrin family: integrin alpha9 in biology and cancer. Adv Biol Regul 52(2): 326-339.

Hsieh YH, Margaret Juliana M, Ho KJ, Kuo HC, van der Heyde H, Elmets C, Chang PL (2011) Host-derived osteopontin maintains an acute inflammatory response to suppress early progression of extrinsic cancer cells. Int J Cancer 131(2): 322-333.

Huang XZ, Wu JF, Ferrando R, Lee JH, Wang YL, Farese Jr RV, Sheppard D (2000) Fatal bilateral chylothorax in mice lacking the integrin alpha9betal. Mol Cell Biol 20(14): 5208-5215.

Kanayama M, Kurotaki D, Morimoto J, Asano T, Matsui Y, Nakayama Y, Saito Y, Ito K, Kimura C, Iwasaki N, Suzuki K, Harada T, Li HM, Uehara J, Miyazaki T, Minami A, Kon S, Uede T (2009) \{alpha\}9 Integrin and its ligands constitute critical joint microenvironments for development of autoimmune arthritis. J Immunol 182(12): 8015-8025.

Majumder M, Tutunea-Fatan E, Xin X, Rodriguez-Torres M, Torres-Garcia J, Wiebe R, Timoshenko AV, Bhattacharjee RN, Chambers AF, Lala PK (2012) Co-expression of alpha9betal integrin and VEGF-D confers lymphatic metastatic ability to a human breast cancer cell line MDA-MB-468LN. PLoS One 7(4): e35094.

Mas-Moruno C, Rechenmacher F, Kessler H (2010) Cilengitide: the first anti-angiogenic small molecule drug candidate design, synthesis and clinical evaluation. Anticancer Agents Med Chem 10(10): 753-768.

Masia A, Almazan-Moga A, Velasco P, Reventos J, Toran N, Sanchez de Toledo J, Roma J, Gallego S (2012) Notch-mediated induction of $\mathrm{N}$-cadherin and alpha9-integrin confers higher invasive phenotype on rhabdomyosarcoma cells. Br J Cancer 107(8): 1374-1383.

Matsui T, Tamaya K, Seki E, Osajima K, Matsumoto K, Kawasaki T (2002a) Val-Tyr as a natural antihypertensive dipeptide can be absorbed into the human circulatory blood system. Clin Exp Pharmacol Physiol 29(3): 204-208.

Matsui T, Yukiyoshi A, Doi S, Sugimoto H, Yamada H, Matsumoto K (2002b) Gastrointestinal enzyme production of bioactive peptides from royal jelly protein and their antihypertensive ability in SHR. J Nutr Biochem 13(2): $80-86$.

Niland S, Eble JA (2011) Integrin-mediated cell-matrix interaction in physiological and pathological blood vessel formation. J Oncol 2012: 125278

Reardon DA, Nabors LB, Stupp R, Mikkelsen T (2008) Cilengitide: an integrin-targeting arginine-glycine-aspartic acid peptide with promising activity for glioblastoma multiforme. Expert Opin Investig Drugs 17(8): 1225-1235.

Renukuntla J, Vadlapudi AD, Patel A, Boddu SH, Mitra AK (2013) Approaches for enhancing oral bioavailability of peptides and proteins. Int J Pharm 447(1-2): 75-93.

Rittling SR (2011) Osteopontin in macrophage function. Expert Rev Mol Med 13: e15.

Rittling SR, Chambers AF (2004) Role of osteopontin in tumour progression. Br J Cancer 90(10): 1877-1881.
Rittling SR, Matsumoto HN, McKee MD, Nanci A, An XR, Novick KE, Kowalski AJ, Noda M, Denhardt DT (1998) Mice lacking osteopontin show normal development and bone structure but display altered osteoclast formation in vitro. J Bone MinerRes 13(7): 1101-1111.

Roberts PR, Burney JD, Black KW, Zaloga GP (1999) Effect of chain length on absorption of biologically active peptides from the gastrointestinal tract. Digestion 60(4): 332-337.

Scaringi C, Minniti G, Caporello P, Enrici RM (2012) Integrin inhibitor cilengitide for the treatment of glioblastoma: a brief overview of current clinical results. Anticancer Res 32(10): 4213-4223.

Schack L, Lange A, Kelsen J, Agnholt J, Christensen B, Petersen TE, Sorensen ES (2009) Considerable variation in the concentration of osteopontin in human milk, bovine milk, and infant formulas. J Dairy Sci 92(11): 5378-5385.

Senger DR, Asch BB, Smith BD, Perruzzi CA, Dvorak HF (1983) A secreted phosphoprotein marker for neoplastic transformation of both epithelial and fibroblastic cells. Nature 302(5910): 714-715.

Senger DR, Perruzzi CA, Papadopoulos A, Tenen DG (1989) Purification of a human milk protein closely similar to tumor-secreted phosphoproteins and osteopontin. Biochim Biophys Acta 996(1-2): 43-48.

Smith LL, Cheung HK, Ling LE, Chen J, Sheppard D, Pytela R, Giachelli CM (1996) Osteopontin N-terminal domain contains a cryptic adhesive sequence recognized by $\alpha_{9} \beta_{1}$ integrin. J Biol Chem 271(45): 28485-28491.

Staniszewska I, Zaveri S, Del Valle L, Oliva I, Rothman VL, Croul SE, Roberts DD, Mosher DF, Tuszynski GP, Marcinkiewicz C (2007) Interaction of alpha9betal integrin with thrombospondin-1 promotes angiogenesis. Circ Res 100(9): 1308-1316.

Uede T (2011) Osteopontin, intrinsic tissue regulator of intractable inflammatory diseases. Pathol Int 61(5): 265-280.

Vantyghem SA, Allan AL, Postenka CO, Al-Katib W, Keeney M, Tuck AB, Chambers AF (2005) A new model for lymphatic metastasis: development of a variant of the MDA-MB-468 human breast cancer cell line that aggressively metastasizes to lymph nodes. Clin Exp Metastasis 22(4): 351-361.

Vermeirssen V, Van Camp J, Verstraete W (2004) Bioavailability of angiotensin I converting enzyme inhibitory peptides. Br J Nutr 92(3): 357-366.

Vlahakis NE, Young BA, Atakilit A, Hawkridge AE, Issaka RB, Boudreau N, Sheppard D (2007) Integrin alpha9betal directly binds to vascular endothelial growth factor (VEGF)-A and contributes to VEGF-A-induced angiogenesis. J Biol Chem 282(20): 15187-15196.

Walsh EM, Kim R, Del Valle L, Weaver M, Sheffield J, Lazarovici P, Marcinkiewicz C (2012) Importance of interaction between nerve growth factor and alpha9betal integrin in glial tumor angiogenesis. Neuro-oncol 14(7): 890-901.

Weidner N, Semple JP, Welch WR, Folkman J (1991) Tumor angiogenesis and metastasis-correlation in invasive breast carcinoma. N Engl J Med 324(1): $1-8$.

Weis SM, Cheresh DA (2011) Alphav integrins in angiogenesis and cancer. Cold Spring Harb Perspect Med 1(1): a006478.

Weisshardt P, Trarbach T, Durig J, Paul A, Reis H, Tilki D, Miroschnik I, Ergun S, Klein D (2012) Tumor vessel stabilization and remodeling by anti-angiogenic therapy with bevacizumab. Histochem Cell Biol 137(3): 391-401.

Wickham M, Faulks R, Mills C (2009) In vitro digestion methods for assessing the effect of food structure on allergen breakdown. Mol Nutr Food Res 53(8): 952-958.

Wu Y, Denhardt DT, Rittling SR (2000) Osteopontin is required for full expression of the transformed phenotype by the ras oncogene. Br J Cancer 83(2): 156-163.

Yamada S, Bu XY, Khankaldyyan V, Gonzales-Gomez I, McComb JG, Laug WE (2006) Effect of the angiogenesis inhibitor Cilengitide (EMD 121974) on glioblastoma growth in nude mice. Neurosurgery 59(6): 1304-1312.

Yokosaki Y, Tanaka K, Higashikawa F, Yamashita K, Eboshida A (2005) Distinct structural requirements for binding of the integrins alphavbeta6, alphavbeta3, alphavbeta5, alpha5betal and alpha9betal to osteopontin. Matrix Biol 24(6): 418-427.

Zaloga GP, Siddiqui RA (2004) Biologically active dietary peptides. Mini Rev Med Chem 4(8): 815-821.

This work is published under the standard license to publish agreement. After 12 months the work will become freely available and the license terms will switch to a Creative Commons AttributionNonCommercial-Share Alike 3.0 Unported License. 